\title{
INFLUENCING THE INDENTATION CURVES BY THE BLUNTNESS OF THE BERKOVICH INDENTER AT THE FEM MODELLING
}

\author{
Jaroslav Ková: $\check{R}^{a, *}$, Vladimír FUis ${ }^{a, b}$, JAN TOMÁŠTíí ${ }^{c}$ \\ a Brno University of Technology, Institute of Solid Mechanics, Mechatronics and Biomechanics, Technická \\ 2896/2, 61669 Brno, Czech Republic \\ ${ }^{b}$ Czech Academy of Sciences, Centre of Mechatronics, Institute of Thermomechanics, Technická 2896/2, 616 69 \\ Brno, Czech Republic \\ ${ }^{c}$ Czech Academy of Sciences, Institute of Physics, Joint Laboratory of Optics of Palacky University Olomouc \\ and Institute of Physics of the AS, Na Slovance 1999/2, 18200 Praha 8, Czech Republic \\ * corresponding author: jaroslav.kovar@vut.cz
}

\begin{abstract}
The influence of the Berkovich indenter bluntness on the indentation curves at nanoindentation test of fused silica is determined by the FEM (finite element method) in this paper. The Berkovich indenter, which is mostly used for the nanoindentation test, was assumed for calculations. The indenter is always blunted in real tests. The bluntness of the indenter has an influence on the results of the nanoindentation test. The combination of the nanoindentation test and the FE modelling can be used for the calculation of the parameters of fused silica plasticity and estimation of the bluntness of the indenter. In this paper, the FEM was used for calculating of the indentation curves. Two cases of bluntness were assumed. The curves for ideally sharp or blunted indenter were determined. The results of these calculations showed that the indentation curves are dependent on the bluntness of the Berkovich indenter. The greatest relative influence of the nanoindentation curves by the bluntness is in the area of small values of the indenter displacement. The impact of assuming blunted indenter to calculated parameters of plasticity was assessed. It is often difficult to measure the bluntness of the indenter but it has an influence on the results of the nanoindentation test and should be included into calculations to make it more precise.
\end{abstract}

KEYWORDS: Berkovich indenter, bluntness, finite element method, nanoindentation.

\section{INTRODUCTION}

The nanoindentation test is often used for the testing of materials which cannot be tested by conventional tests. It is often used for the determination of the material parameters of the thin layers or plasticity of the fused silica 1 . The results of the nanoindentation test are often influenced by many conditions. If the FEM is used for modelling of the nanoindentation test, these conditions should be included to the calculation to get more precise results. It was proved that the bluntness of the indenter has a significant influence on the results and it should be assessed to get accurate results especially when the displacements of the indenter are similar like height of the blunted part of the indenter. Because it is very difficult to measure the real shape of the blunted indenter and use it for FEM calculations, it is often modelled like a sphere with known radius but it is unknown if other edges near the tip should be rounded too. In this paper two types of the roundness of the tip of the Berkovich indenter were assessed with four radii of the roundness, the FEM calculations were done for every case, the results were compared and the best case was chosen.

\section{NANOindentation test}

\subsection{NANOINDENTATION OF THE FUSED SILICA}

The nanoindentation test can be used for the testing of the material properties of the fused silica. Unlike the tension test, in which there is not any significant plastic deformation and the tested specimen fails by brittle fracture after elastic deformation, if the nanoindentation test is used the plasticity occurs in the small volume near the tip of the indenter [1]. Fused silica is used in the Oliver-Pharr analysis as a material for the determination of the shape function of the Berkovich indenter [2].

There are few difficulties in the FEM modelling, if the fused silica is assumed. Firstly, the mechanism of the plasticity of the fused silica is not known yet. The most often used models of stress - strain behaviour are either bilinear [3] or Drucker-Prager [4. The bilinear model of the material was chosen for calculations because it should be enough accurate for the main aims of this paper. Another problem in the FEM modelling of the fused silica nanoindentation was found by the Oliver and Pharr [5]. The unloading curve should be mostly influenced by the elastic parameters and can be used for the determination of the Young's modulus but if the FEM is used the results show steeper unload- 
ing curve than results obtained by the experiment and it leads to the overestimation of the Young's modulus from FEM. The reason of this error is still unknown.

\subsection{INDENTER}

Many indenters (like Berkovich, spherical, conical etc.) can be used for the nanoindentation [1]. The most used indenter is the Berkovich indenter, which was used in this paper. The indenters with the rounded tip like spherical, show steeper loading part of the indentation curve than indenters with the sharp tips (Berkovich indenter) [5]. If the bluntness of the Berkovich indenter is assumed, the indentation curve is steeper than indentation curve obtained from the calculation with the sharp indenter [ $[$ ]. This difference is significant in some cases and then the bluntness of the indenter should be included into the calculations otherwise the error in the estimated parameters of the plasticity by the FEM could be significant. The two types of the bluntness of the indenter and few radii of the bluntness of the Berkovich indenter will be assessed in this paper and the indentation curves from the FEM will be compared with the indentation curve obtained by the experiment.

\section{Methods}

The FEM was used for the determination of indentation curves.

\subsection{Models of GeOMETRY}

The Berkovich indenter pressing into the specimen from the fused silica was assumed for calculations. The size of the indenter and the specimen was chosen to be much bigger than the displacements of the indenter (Fig. 1). Then the influence of the edge of the specimen on the results is small. Only one sixth of the specimen and the indenter was modelled because there are three planes of symmetry. Then the bluntness of the indenter was modelled. Two types of the bluntness were assumed. In the case A (Fig. 2), the tip of the indenter and the edges of the indenter were blunted. In the case B (Fig. 3) another roundness, with the same radius as in the case $\mathrm{A}$, was applied on the point where intersect the lines at the border of the roundness. For each case of bluntness, four radii of the bluntness were assessed. The values of the roundness were $100 \mathrm{~nm}, 200 \mathrm{~nm}, 400 \mathrm{~nm}$ and $800 \mathrm{~nm}$. To compare the results, the case without bluntness was calculated too.

\subsection{BOUNDARY CONDITIONS}

The indenter was loaded by the displacement of the specimen in two loadsteps. In the first loadstep, the displacement $612 \mathrm{~nm}$ plus the gap between the specimen and the blunted indenter against the way of the $\mathrm{Z}$ axis was applied at the upper area of the indenter. In the second loadstep, the unloading by the displacement of the indenter back to the $200 \mathrm{~nm}$ was done. The displacement of the lower area of the specimen in

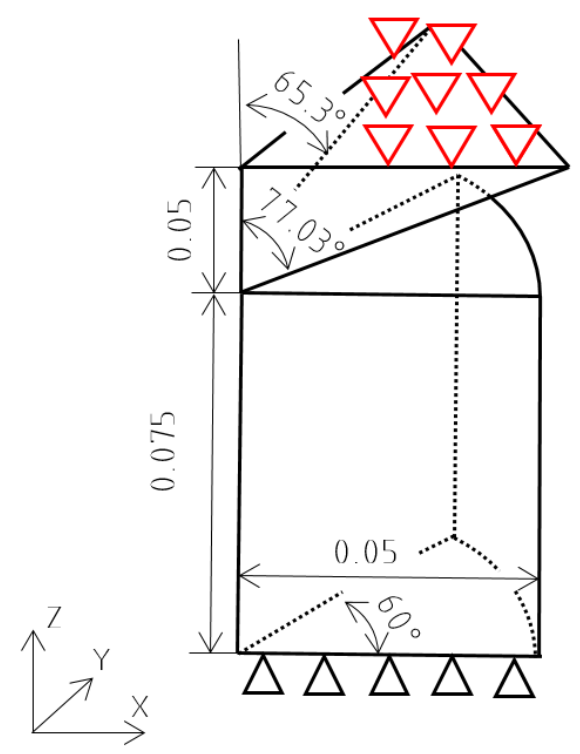

Figure 1. Geometry of the indenter and specimen $[\mathrm{mm}]$.



Figure 2. Mesh of the tip of the indenter with the bluntness $200 \mathrm{~nm}$, (case A, red line shows the border of the blunted area, yellow the border between the blunted tip and the edge).

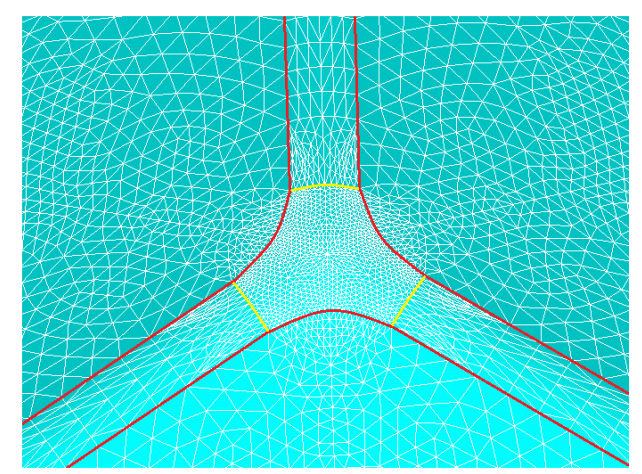

Figure 3. Mesh of the tip of the indenter with the bluntness $200 \mathrm{~nm}$ (case B, red line shows the border of the blunted area, yellow the border between the blunted tip and the edge).

the way of the $\mathrm{Z}$ axis was fixed. On the areas of the symmetry, the displacements perpendicular to these areas were fixed. 


\subsection{Models OF MATERIAL}

The diamond indenter was modelled with the linear elastic model of material with the Young's modulus $\mathrm{E}=1141000 \mathrm{MPa}$ and the Poisson's ratio $\mu=0.07$. The fused silica was modelled with the bilinear model of material. The elastic parameters were taken from [1] $(\mathrm{E}=72000 \mathrm{MPa}, \mu=0.17)$. The parameters of plasticity (yield strength and hardening) were estimated from the experimental data by this procedure. From the comparison of the experimental data and the results from the FEM, the bluntness of the indentor in the case B was determined like $200 \mathrm{~nm}$. The indentation fits well in the area of the low loads where the plasticity is not so important. Then the yield stress was estimated like $\mathrm{R}_{e}=4000 \mathrm{MPa}$. The hardening of the material was not assumed.

\subsection{Mesh and the FEM calculation}

The mesh was made coarse at the edge of the specimen but very fine at the centre to obtain precise results (Fig. 4). Similar procedure was used on the indenter (Fig. 2 and 3). The elements of the type SOLID 185 were used on the specimen and the quadratic SOLID 186 elements on the indenter to describe the edge of the indenter more precise. Between the indenter and the specimen, the contact region was made and CONTA 174 / TARGE 170 elements were used. To obtain more accurate results, the penetration tolerance factor was reduced to 0.01 .

Both loadsteps were divided into a few substeps and the large displacements algorithm was applied. Because of the small values of the displacements, the force convergence criterion was lower to 0.00001 , to obtain more precise results. The calculation with this algorithm was done for every value of the bluntness of the indenter.

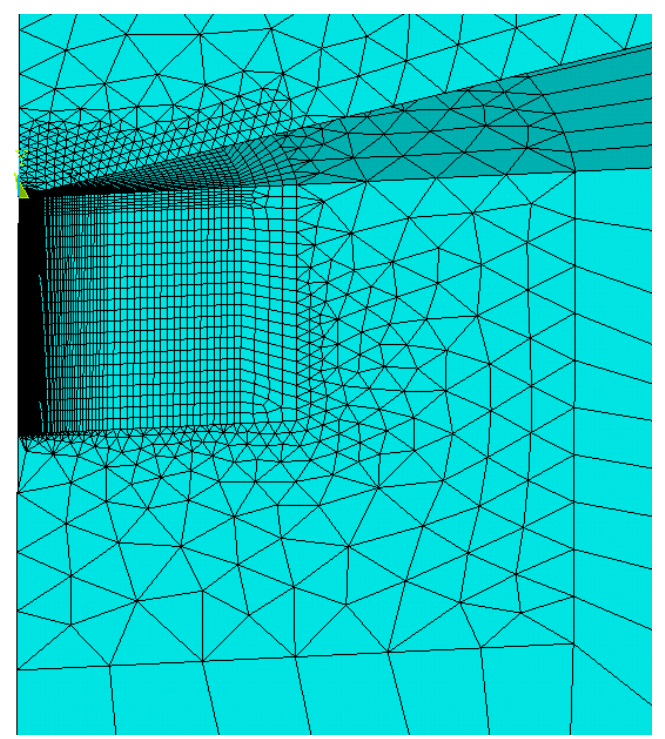

Figure 4. The mesh in the area near contact.

\section{RESULTS AND DISCUSSION}

The indentation curves (Fig. 5) were obtained as the results of the FEM calculations. The curve obtained by the model without shape imperfection showed the least steep loading curve and unloading curve too. The steepness of the curves grew with the bluntness. This agreed with the Oliver-Pharr analysis, where the higher steepness of the unloading curve is taken into account by the indenter shape function. If the sharp indenter was assumed, the unloading curve was still steeper than the experimental. This error was firstly observed by [5] and it is not explained yet. It could be caused by not enough accurate model of material but this was not important for this paper, which was focused on the assessing of the influence of the bluntness of the indenter. The difference between the results with the bluntness A (blunted only the tip and the edges Fig. 2 and the bluntness B (blunted the tip, the edges, and rounded points, Fig. 3) was small, if the bluntness was low but it grew with the growing bluntness. The relative differences of the maximal values of the force related to the bluntness were: $1.54 \%$ for bluntness $100 \mathrm{~nm}, 2.92 \%$ for $200 \mathrm{~nm}$, $5.78 \%$ for $400 \mathrm{~nm}$ and $11.4 \%$ for $800 \mathrm{~nm}$. The bluntness B should be nearer to the reality because the edges should be blunted at least a little but for new indenters, which are not so much blunted, the difference between these models was smaller than $2 \%$. When the bluntness B with radius $200 \mathrm{~nm}$ was assumed, the loading curve obtained from the FEM calculation fitted the curve from the experiment. The similar but a little less accurate results were obtained by the bluntness $A$ with radius of $400 \mathrm{~nm}$.

The bluntness influenced every part of the curve because it shifted the curve except the lower part of the curve which was mostly influenced because there is the spherical part of the tip of the indenter (Fig. 6). The steepness of the curve in this area dramatically grew with the growing bluntness of the indenter. This fact is in the agreement with the results of the indentation curves obtained by the sharp indenters like Berkovich and spherical indenter. The indentation curves obtained by the spherical indenters show steeper indentation curves [6]. From the Fig. 6 is clear that the bluntness B with radius of the $200 \mathrm{~nm}$ was very near to the experimental curve even in the area of the low displacements. The similar relative error showed bluntness A with radius $400 \mathrm{~nm}$. Other cases show bigger relative errors in the area of the small displacements than in the maximal values of the forces. Because the influence of the plasticity is smaller in this area than in the end of loading and the influence of the bluntness is higher, the agreement of the curves from the experiment and the FEM calculation in the area of the small displacements is great criterion for the determination of the bluntness of the indenter for the next calculations. 


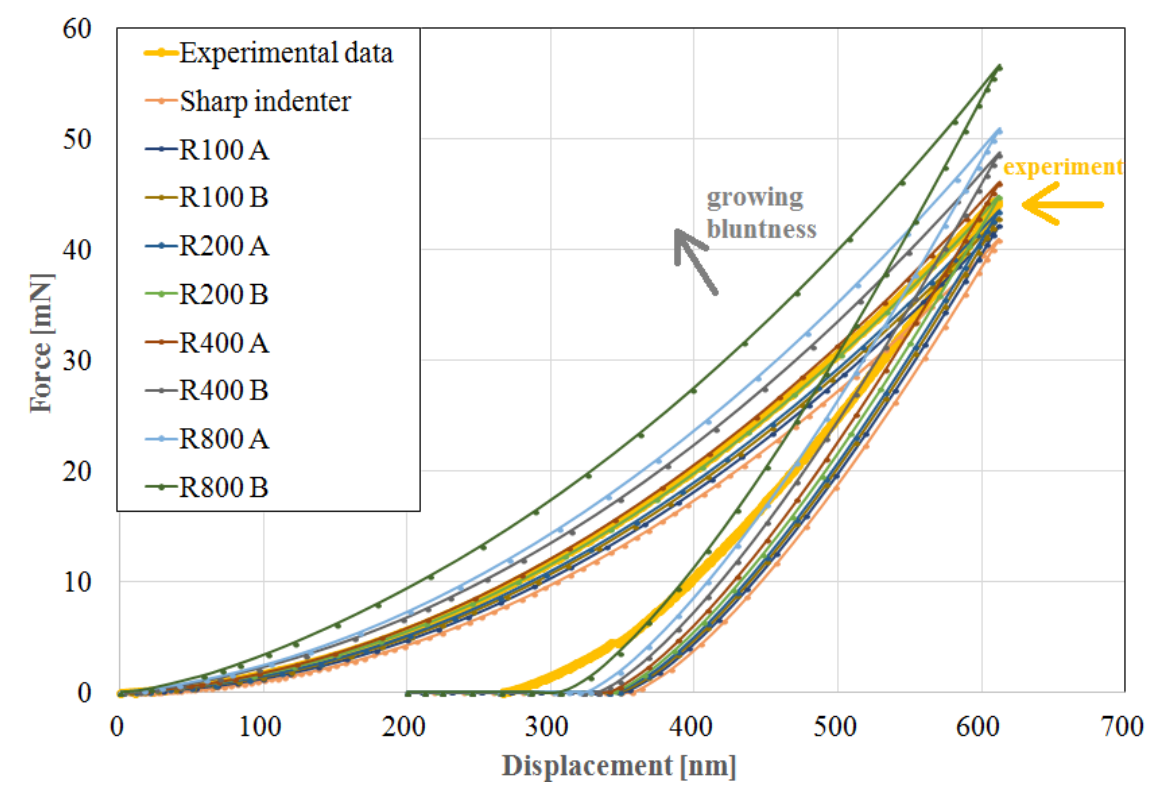

FIGURE 5. Indentation curves for all cases.

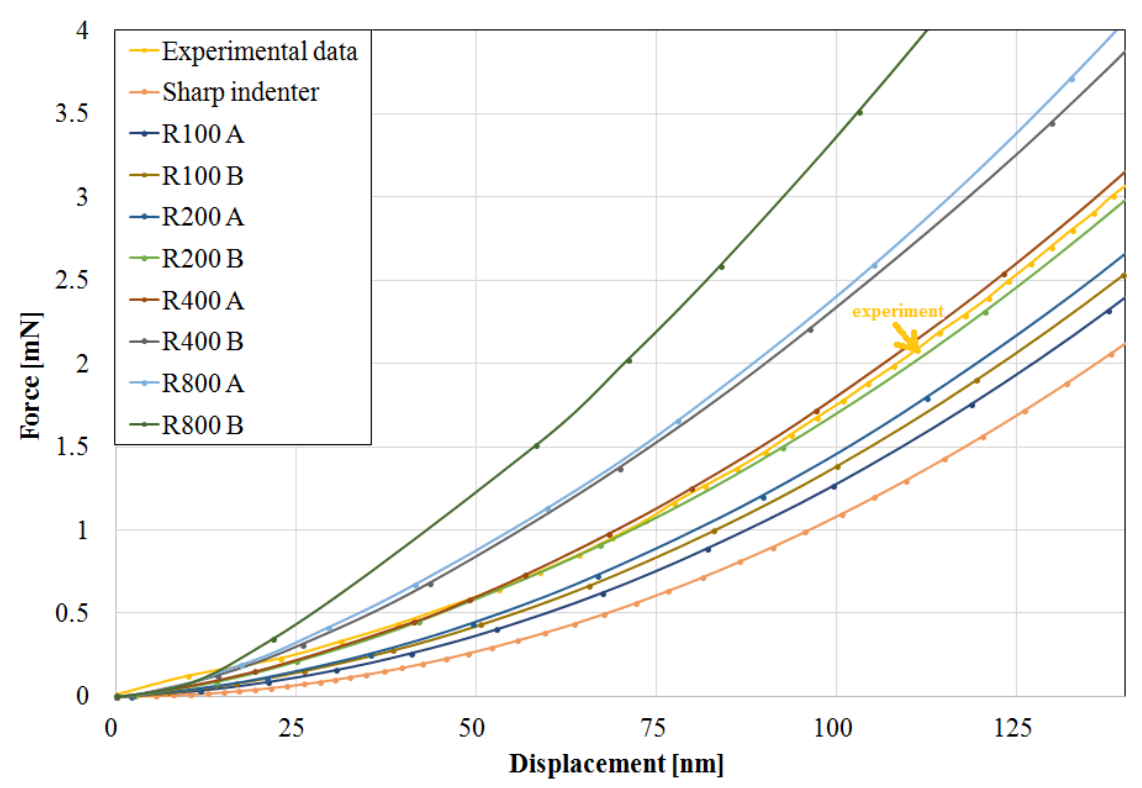

Figure 6. Indentation curves in the area of small displacements (detail of the Fig. 5 .

\section{CONClusions}

The two types of the bluntness of the Berkovich indenter with four radii of the roundness have been compared in this paper. The loading and the unloading curves were getting steeper while the radius of the roundness grew. The bluntness $\mathrm{B}$, in which are the edges of the indenter, the tip of the indenter, and the points, where the edges intersect, blunted, gave steeper indentation curves than bluntness A, in which are blunted only the edges and the tip of the indenter. The indentation curve for the indenter with the bluntness B with radius $200 \mathrm{~nm}$ was the closest to the experimental curve. There still remained the problem with higher steepness of the unloading curve in the results. It could be caused by the inaccuracy of the used model of material. The bluntness had the biggest impact in the area with the small displacements, where the blunted spherical tip caused higher steepness of the indentation curve. Elastic parameters are known and the fitted parameters of the plasticity have only small impact in the area of the small displacements. Then this could be used as a great criterion for the determination of the bluntness of the indenter with the FEM in the future work.

\section{ACKNOWLEDGEMENTS}

The experimental data were measured by the Institute of Physics of the Czech Academy of Sciences, Joint Laboratory of Optics of Palacky University Olomouc and Institute of Physics AS CR. 


\section{REFERENCES}

[1] J. Kovář, V. Fuis. FEM simulation of the nanoindentation test with rigid and non-rigid indenter. Engineering Mechanics 2019 pp. 189-192, 2019. DOI:10.21495/71-0-189

[2] W. Oliver, G. Pharr. An improved technique for determining hardness and elastic modulus using load and displacement sensing indentation experiments. Journal of Materials Research 7(6):1564-1583, 1992. DOI:10.1557/jmr.1992.1564.

[3] D. Torres-Torres, J. Muñoz-Saldaña, L. A. G.-L. de Guevara, et al. Geometry and bluntness tip effects on elastic-plastic behaviour during nanoindentation of fused silica: experimental and FE simulation. Modelling and Simulation in Materials Science and Engineering 18(7):075006, 2010. DOI:10.1088/0965-0393/18/7/075006

[4] K. Gadelrab, F. Bonilla, M. Chiesa. Densification modeling of fused silica under nanoindentation. Journal of Non-Crystalline Solids 358(2):392-398, 2012. DOI:10.1016/j.jnoncrysol.2011.10.011.

[5] S. Shim, W. Oliver, G. Pharr. A comparison of 3d finite element simulations for berkovich and conical indentation of fused silica. International Journal of Surface Science and Engineering 1(2/3):259, 2007. DOI:10.1504/ijsurfse.2007.015028

[6] N. Yu, A. A. Polycarpou, T. F. Conry. Tip-radius effect in finite element modeling of sub-50 nm shallow nanoindentation. Thin Solid Films 450(2):295-303, 2004. DOI:10.1016/j.tsf.2003.10.033. 